\title{
Multilinear principal component analysis for iris biometric system
}

\author{
Chetana Kamlaskar ${ }^{1}$, Aditya Abhyankar ${ }^{2}$ \\ ${ }^{1}$ School of Science and Technology, Y. C. M. Open University, Maharashtra, India \\ ${ }^{2}$ Department of Technology, Savitribai Phule Pune University, Pune, India
}

\begin{tabular}{l}
\hline \hline Article Info \\
\hline Article history: \\
Received Apr 1, 2021 \\
Revised Jul 12, 2021 \\
Accepted Jul 28, 2021 \\
\hline
\end{tabular}

\section{Keywords:}

Feature fusion

Iris biometric

Multilinear principal

component analysis

Multilinear subspace learning

Wavelet packet

decomposition

\begin{abstract}
Iris biometric modality possesses inherent characteristics which make the iris recognition system highly reliable and noninvasive. Nowadays, research in this area is challenging compact template size and fast verification algorithms. Special efforts have been employed to minimize the size of the extracted features without degrading the performance of the iris recognition system. In response, we propose an improved feature fusion approach based on multilinear subspace learning to analyze Iris recognition. This approach consists of four stages. In the first stage, the eye image is segmented to extract the iris region. In the second step, wavelet packet decomposition is conducted to extract features of the iris image, since good time and frequency resolutions can be provided simultaneously by the wavelet packet decomposition. In the next step, all decomposed nodes or packets are arranged as a $3^{\text {rd }}$ order tensor rather than a long vector, in which feature fusion is directly implemented with multilinear principal component analysis (MPCA). This approach provides a more compact or useful low-dimensional representation directly from the original tensorial representation. Finally, a discriminative tensor feature selection mechanism and classification strategy are applied to iris recognition problem. The obtained results indicate the usefulness of MPCA to select discriminative features and fuse them effectively. The experimental results reveal that the proposed tensor-based MPCA approach achieved a competitive matching performance on the SDUMLA-HMT Iris database with an adequate acceptable rate.
\end{abstract}

This is an open access article under the CC BY-SA license.

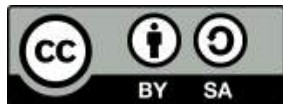

\section{Corresponding Author:}

Chetana Kamlaskar

School of Science and Technology

Y. C. M. Open University, Maharashtra, India

Email: chetana.kamlaskar@gmail.com

\section{INTRODUCTION}

Iris recognition is one of the most trusted biometric technologies in terms of human identification and verification with a wide range of applications, including airport automatic check-in, access systems or humanitarian aid missions, and many more. Compared with face and fingerprint biometric, iris pattern has rich texture information [1] details such as rings, corona, crypts, contraction furrows, ciliary processes, freckles, and colouration. Iris patterns are unique and highly distinctive, and non-invasive as well as highly stable with time. For accurate iris recognition of individuals, the most discriminating information contained in the iris pattern needs to be extracted. Hence, it is crucial to choose a suitable method for feature extraction [2]. More discriminating features can be extracted in a wavelet transform (WT) domain than in a time domain. This work uses significant features extraction based on wavelet packet decomposition (WPD) using Haar wavelet. WPD 
gives reasonably better performance because the dominant frequencies of iris texture are located in the lower and middle frequency channels.

In the first approach of the experiment, energy (E) based criterion is used to select the appropriate wavelet packet after 3-level decomposition. Then using an adaptive threshold, appropriate packet coefficients are quantized into 1,0 or -1 . The feature vector is generated by using the concatenation of quantized coefficients of appropriate energy packets. For the classification of iris recognition system, triangle square ratio similarity measure is used. This approach is implemented just for comparison with other approaches.

This work is mainly focused to propose a new efficient and robust algorithm for compact feature representation and classification of Iris images. The proposed algorithm differs from the existing Iris recognition system at the feature representation and classification stage. Here, in the second approach of the experiment, after 3-level WPD of a normalized iris image, all packets WPD except the first packet which represents DC component, are used to represent the features of the iris image. All of these packets are arranged into a $3^{\text {rd }}$ order tensor rather than a long vector. This $3^{\text {rd }}$ order tensor is further processed by multilinear principal component analysis (MPCA), as MPCA represents multidimensional data as tensors rather than vectors, with three key benefits, preserve the multidimensional structure, lower computational demand, and requires fewer parameters to estimate [3]. So, by using MPCA, the effective components for each feature can be selected and extracted simultaneously, and combined together. These fused components, as a new feature of the iris, is fed to a modified angle distance (MAD) classifier for automated classification. MPCA is proved to be a more effective method for multiple feature fusion and representation. The contribution of our work as follows:

- Utilized MPCA for the discriminative feature selection from WPD tensors of iris images and MAD similarity measure for classification

- The proposed approach create a compact low-dimensional discriminative feature vector and run with minimum computational time.

- The proposed approach is evaluated with receiver operating characteristic (ROC) and equal error rate (EER) on the SDUMLA HMT Iris dataset.

The paper is organized as follows: Section 2 describes related work, the proposed MPCA is outlined in Section 3, the experimental design is presented and the results are discussed in Section 4, and finally, Section 5 concludes the work.

\section{RELATED WORK}

The most successful proven methods for iris recognition include work proposed by Daughman [4]. In order to extract iris features, he makes use of quadrature 2D Gabor wavelets and encodes the iris image to a binary code of 256 bytes (2048 bits) in length, referred as an iris code. Hamming distance is used to indicate the similarity of two iris codes. Iris recognition system proposed by [5] uses Laplacian of Gaussian filters for the decomposition of the iris region. Then, constructed Laplacian pyramid to generate a compact iris template. The similarity between two iris templates is determined using correlation comparison. Boles and Boashash [6] have proposed a system based on dyadic 1D wavelet transform with the zero crossing detectors for iris feature extraction and makes use of two dissimilarity functions for comparison of iris representation. They claim that noise influences can be eliminated with the zero crossing detectors [6].

Zhu et al. [7] employ multi-channel Gabor filtering and the wavelet transform to extract iris feature vector and makes use of weighted Euclidean distance classifier to identify the iris. An iris image is decomposed using 2D Haar wavelet transform by [8]. In this work, 87-bit code feature is generated by quantizing the fourth level high frequency information, and a modified competitive learning neural network (LVQ) is used for classification. Wavelet packet transform (WPT) using Haar wavelet for extraction of iris texture approach is used in [9]. In this study, only suitable sub images are selected by applying WPT decomposition. Then, WPT coefficients of selected sub images are encoded as iris feature vector and compared Manhattan distance between the two corresponding iris vectors for matching.

The approach proposed by Hariprasath and Venkatasubramanian [10] is based on 2D WPT. First, iris region is encoded into a sequence of $2 \mathrm{D}$ wavelet packet coefficients with a size of the feature vector of 1280 bits. Then, exclusively OR comparisons are made between two different iris codes. The approach presented in [11] proposes Iris feature extraction using Haar wavelet on IITD database and Hamming distance matcher to achieve higher verification performance. Recently, biometric authentication proposed by [12] uses continuous curvelet transform combined with PCA for Iris feature extraction. The performance is evaluated with three 
classifiers - k-nearest neighbors (KNN), support vector machine (SVM), neural network (NN) and achieved average recognition rates of $91.0 \%, 93.0 \%$, and $97.0 \%$ respectively. According to these previous studies a wavelet transform is one of the relevant tools to extract the most distinctive features contained in an iris image. Hence, for tensor representation, WPD was chosen which has linear computational complexity.

MPCA based tensor feature extraction has found widespread use in various applications of computer vision and pattern recognition, recent applications include face recognition [13], signal processing, handwriting, digital number recognition, content analysis, anomaly detection in data [14], gait recognition [15]. A new framework of MPCA for dimensionality reduction and feature extraction of the tensor object is proposed by [3] with an application to gait recognition. Motivated by the success of MPCA in feature extraction, in this work, we propose feature fusion using tensor based MPCA for Iris recognition.

\section{THE PROPOSED METHOD}

In general, the iris recognition system consists of four processing modules - Segmentation, Normalisation, Feature extraction and encoding, and Matching. Figure 1 shows the block diagram of the proposed feature fusion method. Here, we aim to effectively perform multiple feature fusion using tensor-based multi-linear subspace learning method.

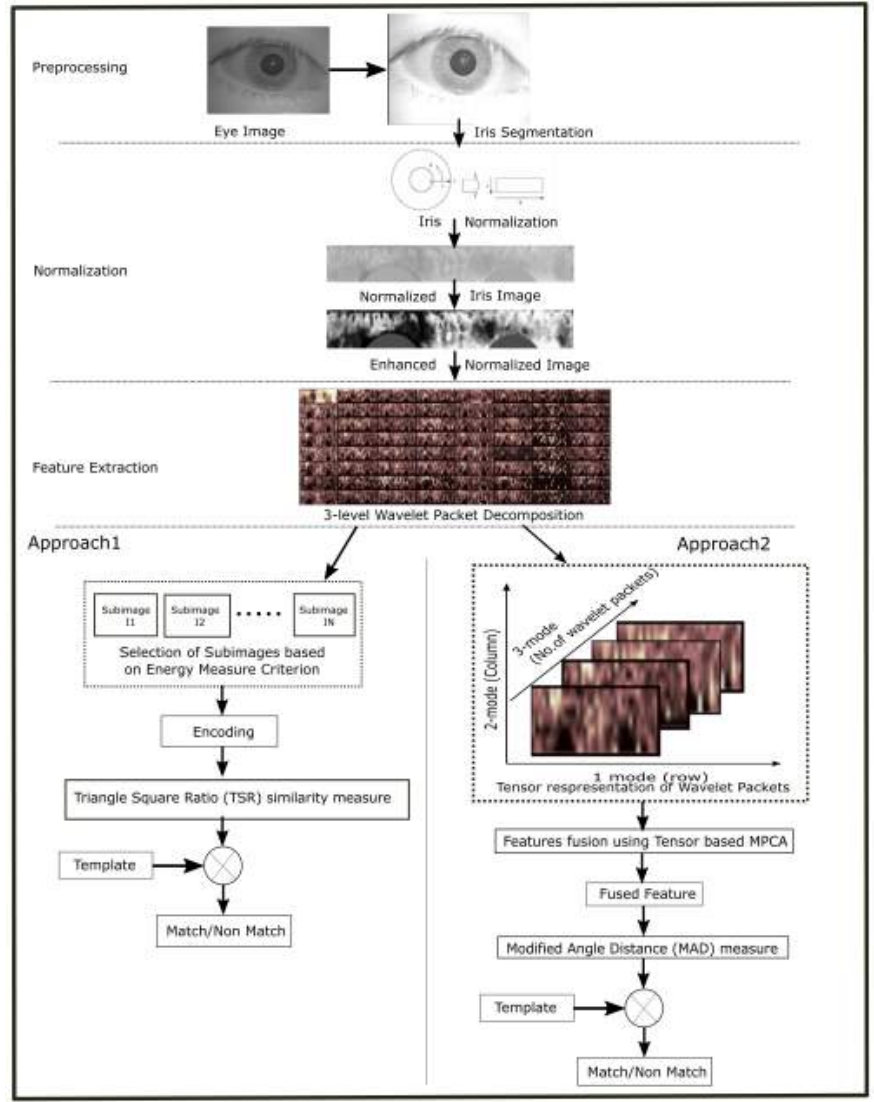

Figure 1. Block diagram of the proposed iris recognition system

\subsection{Preprocessing}

The first step is segmentation, where iris region is isolated from an eye image. This step plays a key role in the recognition performance. As improper segmentation can lead to incorrect feature extraction, illumination normalization is performed prior to iris segmentation [16], [17]. In the next step, normalization is done to transform or map the extracted iris region into a fixed rectangular block as the size of the iris may differ from one eye to another. For this, Daugman's Rubber sheet model [4] is used. In this, each pixel of the isolated iris is remapped to a pair of polar coordinates to make iris representation invariant to the size of iris and pupil 
dilation. Finally, the normalized iris is subjected to feature extraction. Before that, histogram equalization is performed to enhance the quality of normalized iris.

\subsection{Iris feature extraction using WPD}

Only the significant features of the iris pattern must be extracted and encoded for accurate recognition of individuals. In this work, WT is used to extract features from the enhanced iris images. WT analyzes the signal or image at different frequency bands with different resolutions by decomposing it into approximation and detail coefficients. The decomposition of the signal into different frequency bands is obtained simply by successive high pass and low pass filtering of the time domain signal. WT decomposes an image into four subimages or sub-bands such as approximation coefficients (LL), horizontal coefficients (LH), vertical coefficients (HL) and diagonal coefficients (HH). One or more of these sub-bands can be split into smaller sub-bands, which can be split again, and so on. Hence, more discriminating features can be extracted in a WT domain than a time domain. However, WT only displays sufficient frequency resolution at low frequencies but poor frequency resolution at high frequencies.

As an extension of WT, WPD is developed to achieve fine frequency resolution at both low and high frequencies. In WPD, each of approximation and detailed sub-bands are further processed as opposed to WT where only approximation sub-bands are further processed. This results in splitting the whole frequency plane into equally sized bands. Hence, WPD enables us to zoom into desired frequency channels for further decomposition and yield a better representation of signals [9]. For this reason, WPD is more suitable to extract local patterns of each iris at different resolution levels, which contains the main diversities of different irises. In this work, Haar WT was carried out up to 3-level on the enhanced iris images after the normalization step. Haar is the simplest orthogonal wavelet system, compact support in time, has 1 vanishing moment. It provides a simple and computationally efficient approach for analysing the local aspects of a signal, defined by (1) and (2),

$$
\psi(x)=\left\{\begin{array}{l}
1, \quad \text { if } 0 \leq x<\frac{1}{2}, \\
-1, \quad \text { if } \frac{1}{2} \leq x<1, \\
0, \quad \text { otherwise }
\end{array}\right.
$$

and Haar scaling function computes average or approximation.

$$
\phi(x)= \begin{cases}1, & \text { if } 0 \leq x<1 \\ 0, & \text { otherwise }\end{cases}
$$

Figure 2 illustrates the WPD structure after 3-level WPD. With the levels computed from top to bottom, time resolution decreases, whereas frequency resolution increases. A quadtree with 64 output sub-images is generated. The sub-images are referred as packets or nodes that have coefficients of approximation (A), horizontal detail $(\mathrm{H})$, vertical detail (V) and diagonal detail (D).

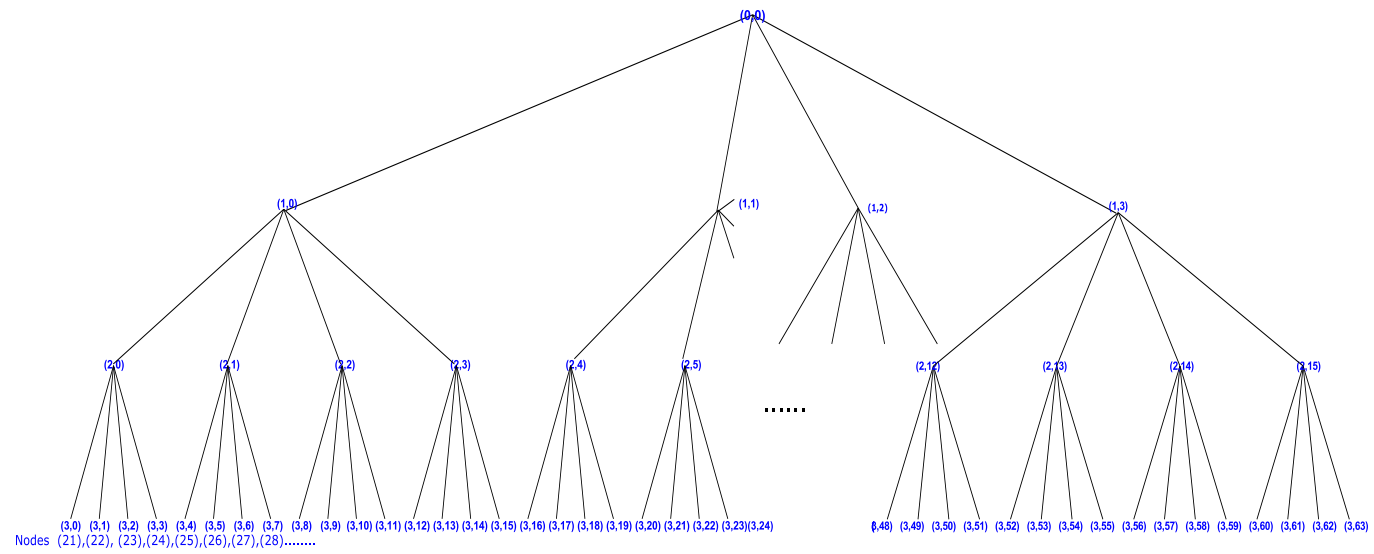

Figure 2. WPD structure for 3-level decomposition 


\subsection{Feature subset selection and vector creation}

In this paper, two approaches are proposed to select the optimal set of features.

Approach 1: Energy Measure based Packet Selection. Energy Measure (E): The energy-based criterion is used to choose useful sub-images for feature encoding as wavelet maxima energy points are capable of detecting sharp variation points, and of formulating a signal the presentation that is well adapted for characterizing patterns. Energy distribution for an iris image $\mathrm{f}(\mathrm{x}, \mathrm{y})$ with $1<x<M$, and $1<y<N$ can be calculated using wavelet packets [18] and energy measure using (3), where $\mathrm{M}$ is number of rows and $\mathrm{N}$ is number of columns of the enhanced normalized iris image.

$$
E=\frac{1}{M N} \sum_{x=1}^{M} \sum_{y=1}^{N}|f(x, y)|^{2}
$$

Figure 3 shows the average energy distribution of 244 different iris images with Haar WPT. It consists of total 64 packets ranging from $(3,0)$ (corresponding to node 21 ) to $(3,63)$ (corresponding to node 84 ) at 3 -level decomposition. It is observed that if the image has distinct features with some frequency and direction, the corresponding sub-images or packets have larger energies in wavelet transform.

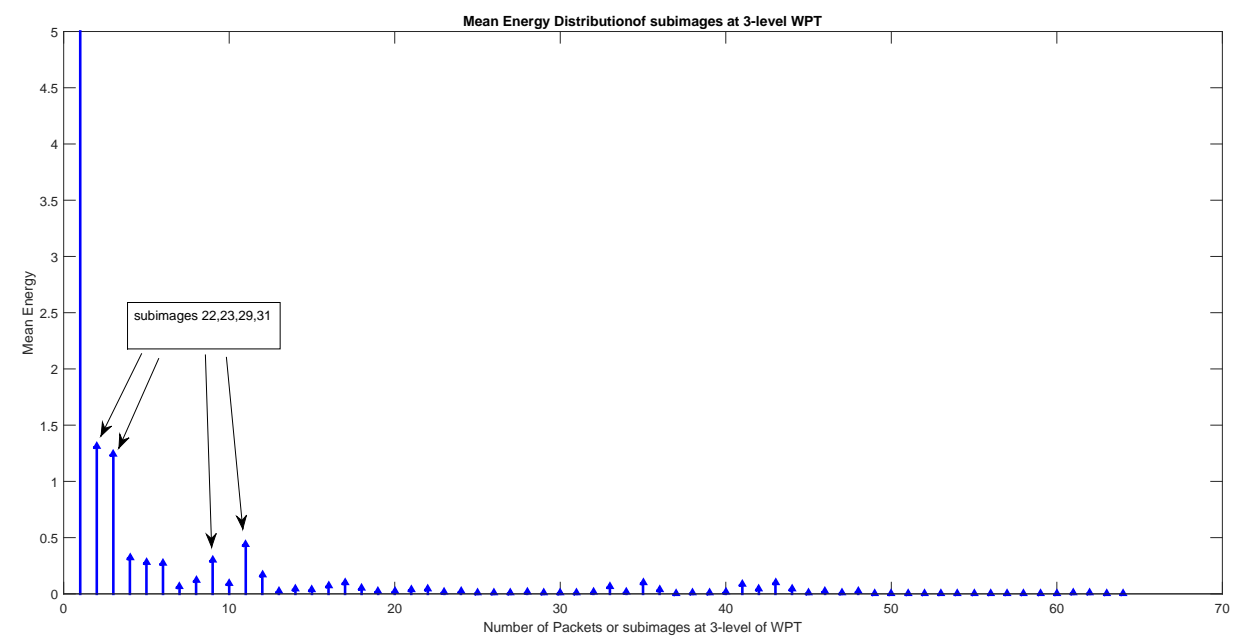

Figure 3. Average energy distribution of each packets

From Figure 3, appropriate dominant energies are chosen to compute iris code. The node 21 corresponds to packet number $(3,0)$ has offset (DC) information hence, not considered. The subimages at node 22, 23,29 , and 31 retain much higher energy than other sub-images so they are chosen as candidates or samples for encoding. During the experiment, a combination of appropriate packets is used for the selection of sub-images, and their coefficients are used to represent the feature vector.

Encoding WPT coefficients: By applying soft threshold (T), an iris feature vector referred as Iriscode is achieved by quantizing the coefficients into one data element as (4),

$$
F_{i j}=\left\{\begin{array}{l}
1, \quad \text { if } C_{i j}>T, \\
-1, \quad \text { if } C_{i j}<-T, \\
0, \quad \text { otherwise }
\end{array}\right.
$$

where $C_{i j}$ the coefficient of subimage, $\mathrm{T}$ is a soft threshold and $F_{i j}$ is encoded coefficients of that subimage. In this experiment, $T=3 \sigma$, which is more practical in engineering applications [9]. Here, $\sigma$ is the standard deviation of the highest frequency sub-image coefficients, packet number $(3,63)$, that is, node 84 . For enhanced normalized image size of 50x270, after 3-level wavelet packets decomposition, the size of subimage at level 3 is $7 \times 34$ pixels. So every single subimage generates a code of length 238 . If the combination of $N$ subimages is used then it would generate a code of length $\mathrm{Nx} 238$. 
Approach 2: MPCA. After extracting wavelet coefficients at 3-level decomposition of the enhanced normalized iris image, we aim to fuse all of the features effectively to classify iris images. In this work, MPCA, a tensor-based multi-linear subspace learning method is proposed to perform the multiple-feature fusion. It can effectively combine and select all of the features extracted from the original image and consider the interrelationship among different wavelet packet coefficients, without reshaping tensors into vectors. A concept of 'tensor' is introduced to arrange all of the features of one normalized iris image. This work is motivated by multilinear feature extraction methods presented in [3], the MPCA. Here, we propose a novel tensor based feature fusion approach using MPCA to select and combine extracted iris features after wavelet packet decomposition.

\subsubsection{Tensor notations and representation}

A tensor is a N-way array or a multidimensional array [14], and the order of a tensor is the number of dimensions, also known as ways or modes. In this paper, we denote scalars by lower-case letters $(x, y, \ldots)$, vectors (one-way array) by boldface letters $(\mathbf{x}, \mathbf{y}, \ldots)$, matrices (two-way array) by boldface capital letters $(\mathbf{X}, \mathbf{Y}, \ldots)$, and tensors of order three or higher(three-way or higher array) by calligraphic capital letters $(\mathcal{X}, Y, \ldots)$. An $\mathrm{N}^{t h}$ order tensor is denoted as, $\mathcal{X} \in \mathbb{R}^{I_{1} \times I_{2} \ldots \times I_{N}}$. A tensor of $\mathrm{N}^{t h}$ order contains $\mathrm{N}$ indices $i_{n}$, where $n=1, \ldots, N$, and each of which corresponds to the n-mode of $\mathcal{X}$. For example, in this study, size of the normalized iris is 50x270 pixels after 3-level wavelet packets decomposition, the size of subimage or packet at level 3 is $7 \times 34$ pixels. Total 63 packets excluding the first one (DC component) are used together for feature fusion. So, the normalized texture can be represented as $7 \times 34 \times 63$ three-dimensional tensor objects $\left(3^{\text {rd }}\right.$ order tensor) with column, row, and number of wavelet packets respectively. The n-mode product of a tensor $\mathcal{X} \in \mathbb{R}^{I_{1} \times I_{2} \ldots \times I_{N}}$ with a matrix $\mathbf{U} \in \mathbb{R}^{J_{n} \times I_{n}}$ denoted as $\mathcal{X} \mathbf{x}_{n} \mathbf{U}$, is a tensor with entries [14].

$$
\left(\mathcal{X} \mathbf{x}_{n} \mathbf{U}\right)\left(i_{1}, \ldots, i_{n-1}, j_{n}, i_{n+1}, \ldots, i_{N}\right)=\sum_{i_{n}} \mathcal{X}\left(i_{1}, \ldots, i_{n}\right) \cdot \mathbf{U}\left(j_{n}, i_{n}\right)
$$

\subsubsection{MPCA}

In pattern recognition, a tensor object is usually defined in high dimensional tensor space. Recognition methods serving directly on this space have the curse of dimensionality problem and many classifiers behave inadequately given a small number of training samples. Further, handling high dimensional samples are computationally costly. To deal with this and learn features, directly from tensor without reshaping tensors into vectors, researchers have attempted multilinear subspace learning [19]. The PCA is mainly used to reduce data dimension and retain information that characterizes the variation of data as much as possible. However, conventional PCA was originally proposed to process 1-D vectors, which requires all input data to be converted into 1-D vectors before analysis. This unfolding process breaks the natural structure of the input data and loses compact or valuable representations in the original form [14]. PCA also suffers from a small sample problem when the dimension of the unfolded data is much larger than the number of samples. To overcome these difficulties, MPCA is proposed by extending the conventional linear PCA based on multilinear algebra.

The proposed MPCA is a multilinear algorithm performing dimensionality reduction in all tensor modes seeking those bases in each mode that allow projected tensors to capture most of the variation present in the original tensors. The core of the MPCA algorithm [3] is the eigen-decomposition in each mode so the distribution of the eigenvalues is expected to impact significantly on the performance of the algorithm. MPCA has been introduced in detail in [3]. Let, a set of $\mathrm{M}$ tensor objects $\left\{\mathcal{X}_{1}, \mathcal{X}_{2}, \ldots, \mathcal{X}_{M}\right\}$ is available for training with zero mean. Each tensor sample $\mathcal{X}_{m} \in \mathbb{R}^{I_{1} \times I_{2} \ldots \times I_{N}}$ assumes values in a tensor space $\mathbb{R}^{I_{1}} \otimes \mathbb{R}^{I_{2}} \cdots \otimes \mathbb{R}^{I_{N}}$, which is the tensor product of $\mathrm{N}$ vector spaces $\mathbb{R}^{I_{1}}, \mathbb{R}^{I_{2}}, \ldots, \mathbb{R}^{I_{N}}$. The MPCA objective is the determination of $\mathrm{N}$ projection matrices $\left\{\mathbf{U}^{(n)} \in \mathbb{R}^{I_{n} \times P_{n}}, n=1, \ldots, N\right\}$ to map original tensor set $\left\{\mathcal{X}_{m} \in \mathbb{R}^{I_{1} \times I_{2} \ldots \times I_{N}}\right\}_{m=1}^{M}$ into a tensor subspace $\left\{\mathcal{Y}_{m} \in \mathbb{R}^{P_{1} \times P_{2} \ldots \times P_{N}}\right\}_{m=1}^{M}$ with $P_{n} \leq I_{n}, n=1, \ldots, N$ the dimensionality of the projected space is much lower than the original tensor space. Mathematically $[3,14]$,

$$
\mathcal{Y}_{m}=\mathcal{X}_{m} \mathbf{x}_{1} \mathbf{U}^{(1)^{T}} \mathbf{x}_{2} \mathbf{U}^{(2)^{T}} \ldots \mathbf{x}_{N} \mathbf{U}^{(N)^{T}} \in \mathbb{R}^{P_{1} \times P_{2} \ldots \times P_{N}}, m=1, \ldots, M
$$

The feature tensor after projection is obtained as $\left\{\mathcal{Y}_{m}\right\}$ which captures most of the variation in original tensor set, and variations are measured by the total tensor scatter, and $\mathbf{U}^{(n)}$ is the mode-n projection matrix. However, it is hard to obtain all $\mathrm{N}$ projection matrices simultaneously. For this purpose, the alternating least square (ALS) algorithm can be used to solve the optimization of projection matrices approximately [3]. Thus, MPCA formulation is the estimation of the $\mathrm{N}$ projection matrices that maximize the total tensor scatter $\Psi_{T y}$ as (7), 


$$
\left\{\mathbf{U}^{(n)} \in \mathbb{R}^{I_{n} \times P_{n}}\right\}_{n=1}^{N}=\underset{\left\{\mathbf{U}^{(n)}\right\}}{\arg \max } \Psi_{T y}
$$

where $\Psi_{T y}=\sum_{m=1}^{M}\left\|\mathcal{Y}_{m}-\overline{\mathcal{Y}}\right\|_{F}^{2}, \overline{\mathcal{Y}}$ denotes the mean of projected tensor feature calculated as $\overline{\mathcal{Y}}=\frac{1}{M} \sum_{m=1}^{M} \mathcal{Y}_{m}$.

During testing, a test tensor sample $\mathcal{X}$ is first centered by subtracting the mean $\overline{\mathcal{X}}$ obtained from the training data and then projected using (8) to the MPCA features. For the implementation of MPCA algorithm, we referred to the code available at MathWorks.

$$
\mathcal{Y}=(\mathcal{X}-\overline{\mathcal{X}}) \mathrm{x}_{1} \mathbf{U}^{(1)^{T}} \mathbf{x}_{2} \mathbf{U}^{(2)^{T}} \ldots \mathbf{x}_{N} \mathbf{U}^{(N)^{T}}
$$

\subsection{Classification or matcher stage}

After the discriminant features are extracted, the final step of iris recognition is to design a robust matcher. The function of this step is to measure how similar or different templates are and to decide whether they belong to the same person or not. In the training phase, iris code is generated using extracted features for each iris image and stored as a template in the gallery. During the testing phase, iris code of the query sample is compared using different distance measures. The result of this computation is then used as the score of match, with smaller values indicating better matches.

For Approach 1: The most popular distance measures are Euclidean, Manhattan and Cosine distance. Euclidean and Manhattan distance ignore the correlation which is important for measuring the similarity of two vectors while Cosine measures the correlation but ignores the distance between two vectors. To take into account, both, the distance and the correlations between two vectors, triangle square ratio similarity measure [20] is used, defined as (9),

$$
\operatorname{TSR}(a, b)=\frac{\|a-b\|^{2}}{\|a\|^{2}+\|b\|^{2}}=1-\left(\frac{2\|a\|\|b\|}{\|a\|^{2}+\|b\|^{2}}\right) \cos \theta
$$

if $\mathrm{a}$ and $\mathrm{b}$ are unit vectors, then the triangle square ratio is equivalent to Euclidean distance, while $\|a\|=\|b\|$, it is equivalent to the cosine criterion [20].

For Approach 2: The performance of tensor based feature fusion is measured using a Modified angle distance (MAD) similarity measure [3]. It is weighted versions of the cosine angle, defined as (10),

$$
M A D(a, b)=-\frac{\sum_{i=1}^{N} a_{i} \cdot b_{i} / w_{i}}{\sum_{i=1}^{N} a_{i}^{2} \sum_{i=1}^{N} b_{i}^{2}}
$$

where $\mathrm{N}$ is the length of feature vector and $\mathrm{w}$ is weight vector.

\section{RESULTS AND DISCUSSION}

\subsection{Experiment on SDUMLA-HMT multimodal database}

The experiment is performed on the SDUMLA-HMT Multimodal Database from the Group of Machine Learning and Applications, Shandong University [21]. This multimodal data set is a comprehensive collection of five biometric modalities such as face, finger vein, gait, iris and fingerprint of the same subject and it consists of a total 106 subjects. We have chosen iris modality for testing the proposed algorithms. The details of iris modality are mentioned in Table 1 and representative images are shown in Figure 4.

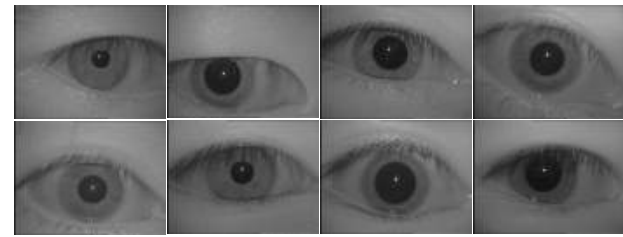

Figure 4. Representative iris images from SDUMLA database 
Table 1. Iris SDUMLA-HMT database

\begin{tabular}{cccc}
\hline Image Resolution & (Iris x impressions per Iris) & Total Images & Format \\
\hline $768 \times 576$ & Left Iris $106 \times 5=530$ & 1060 & 256 gray level BMP \\
& Right Iris $106 \times 5=530$ & & \\
\hline
\end{tabular}

\subsection{Proposed implementation}

In the pre-processing stage, the iris needs to be isolated from an eye image and then normalized to the same size. Prior to this, video-based automatic system for iris recognition (VASIR) [22] is used to analyze the iris image quality. From the quality analysis, it is observed that iris images have very low contrast between sclera and iris, and hence fail to segment iris correctly. So, iris image contrast enhancement is performed using 'imadjust' and log transformation. Iris images are segmented and then normalized using the method proposed in [23]. That means, the iris region is mapped into fixed dimensions of $50(\mathrm{r}) \times 270(\theta)$ of polar image coordinates. The normalized image has low contrast, so adaptive histogram equalization is performed on the image to adjust the contrast. In the feature extraction stage, 3-level wavelet-packet decomposition is used to extract WP coefficients as iris features.

In experiment 1, after 3-level Haar WPD of normalized iris, the size of subimage at level 3 is $7 \times 34$ pixels. So, every single subimage generates an iris code of length 238. Based on the Energy measure criterion, $\mathrm{N}$ numbers of subimages are selected. The resultant feature vector is the concatenation of quantized coefficients of selected subimages. If a combination of $\mathrm{N}$ subimages is used then it generates iris code of length $\mathrm{Nx} 238$. The performance of different combinations of subimages is shown in Table 2. In the matching stage, NN classifier with a triangle square ratio is used.

In experiment 2, a tensor model is built in the feature extraction process. To formulate a tensor representation of each enhanced normalized image of size 50x270, after 3-level Haar WPD, all 63 subimages (each of size 7x34) excluding the first one (DC component) are arranged as a three way tensor as $\mathcal{X} \in \mathbb{R}^{7 \times 34 \times 63}$. Here, MPCA is introduced, which is a tensor based dimensionality reduction algorithm. Then a novel MPCAMAD matcher is constructed which takes advantage of tensor feature extraction and at the same time solves the problem of formation of more discriminative single feature vector by fusing 3-level WPD subimages coefficients.

\subsection{Performance evaluation}

The performance of the system is reported using ROC curve and EER. The ROC curve is the percentage of genuine attempts accepted (i.e. 1-false rejection rate (FRR)) on the y-axis, against the percentage of impostor attempts accepted (i.e. false acceptance rate (FAR)) on the X-axis. The ROC curve measures the accuracy of the matching process for different threshold values and shows the overall performance of the designed system. FAR is referred as the probability that imposter sample is taken as genuine while FRR is referred as the probability that a genuine sample is taken as an imposter one. On the ROC curve, the point where FAR is equal to FRR is referred as EER. The obtained EER value is used to evaluate the recognition accuracy in our experiments. In biometrics, the lower value of EER shows the better recognition performance of the system.

In this work, out of 106 subjects, only 61 subjects are selected based on correct segmentation of iris. We use first 4 images per subject in the training set (61 Classes $\mathrm{x} 4$ impressions per Class) and the remaining for testing. In our experiments, match scores are calculated by comparing every single image with the remaining images. The match scores are divided into inter-class and intra-class matching to justify the effectiveness of the proposed method.

The total number of intra-class comparisons is 366 and that of inter-class comparisons is 29280 . Table 2 shows that by selecting the appropriate combination of subimages to encode iris features, the performance of the recognition system can be improved. The combination results in increasing the length of bit code and provides more texture information from different subimages. If a subimage having high-frequency noisy energy gets combined, it results in degrading the performance. The best EER performance of $2.2985 \%$ is obtained for iris code length of 952 using subimages 22, 23, 29, and 31 of Haar WPT. The ROC curves and EER for various bit lengths of iriscode using Haar WPT are shown in Figure 5. 
Table 2. EER performance for different combinations of subimages of HAAR WPT

\begin{tabular}{lccccc}
\hline $\begin{array}{l}\text { Subimages } \\
\text { Encoded }\end{array}$ & $\begin{array}{c}\text { Feature } \\
\text { (Code length) }\end{array}$ & $\begin{array}{c}\text { Genuine }(\mathrm{G}) \\
\text { Trials }\end{array}$ & $\begin{array}{c}\text { Imposter (I) } \\
\text { Trials }\end{array}$ & $\begin{array}{c}\text { Matcher } \\
\text { (Similarity Measure) }\end{array}$ & EER\% \\
\hline 22,23 & 476 & 366 & 29280 & Triangle & 2.6981 \\
22,31 & 476 & & & square & 5.6660 \\
23,31 & 476 & & & ratio & 2.4556 \\
$22,23,31$ & 714 & & & & 2.4624 \\
$22,23,29$ & 714 & & & & 3.1523 \\
$23,29,31$ & 714 & & & & 2.7322 \\
$22,23,29,31$ & 952 & & & & 2.2985 \\
\hline
\end{tabular}

[1]Training Images: No. of Class $(\mathrm{N})=61$ and Images per Class $(\mathrm{t})=4$

[2] Genuine Trials $(\mathrm{G})=N * t *(t-1) / 2=366, \quad$ [3] Imposter Trials $(\mathrm{I})=N *(N-1) * t * t / 2=29280$

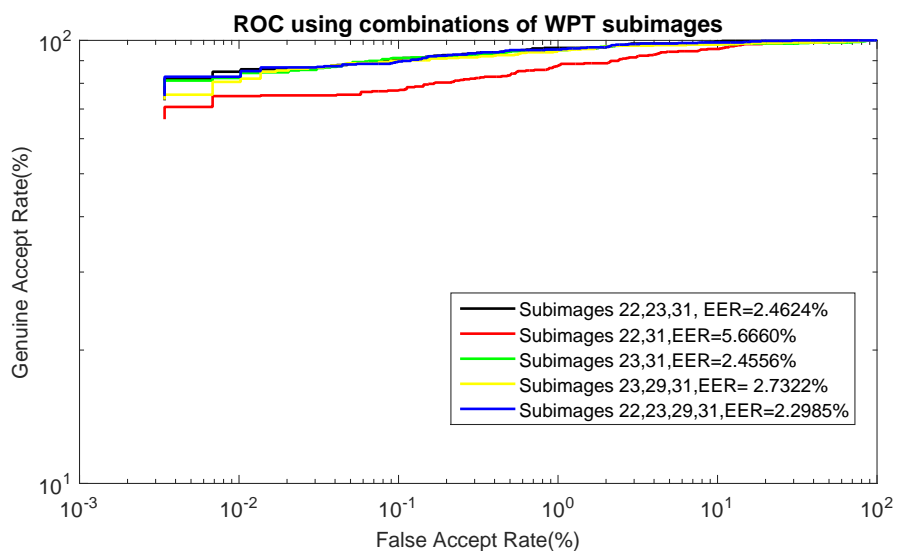

Figure 5. ROC curve for Haar WPT

The result of the proposed tensor based MPCA feature fusion is shown in Figure 6 and Table 3. Here, the performance in terms of EER is tested by varying the number of eigenvectors which represents the dimension of iris features. Using MPCA, the best performance or the lowest EER of $2.2814 \%$ is obtained for feature vector length of 500 and for the remaining feature dimension, EER is slightly high. For comparison purposes, the performance of the tensor based MPCA approach is also investigated for the same bit length of iris code obtained using approach 1 . It is seen that the best performance can be obtained by considering the tensor representation of all 3-level subimages of WPT and then performing multilinear subspace learning feature fusion using MPCA. Thus, approach 2 has the potential to fuse wavelet packet features of iris into a single feature vector which is a highly discriminative fused feature with fewer dimensions. Figure 6 shows the ROC curves of MPCA based feature fusion approach. Thus the proposed tensor-based MPCA approach not only brings the effect of dimension reduction but also significantly outperforms.

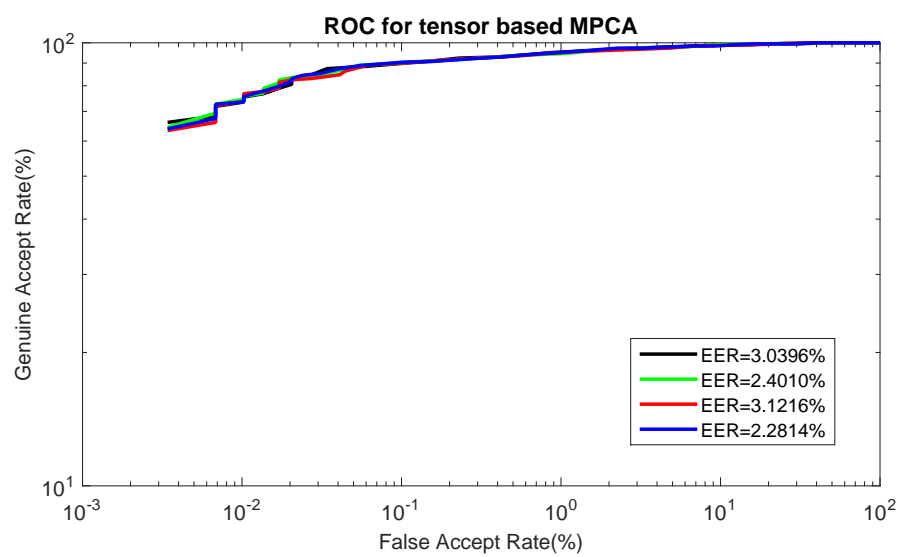

Figure 6. ROC curve for tensor based MPCA 
Table 3. Performance analysis for different feature dimension of MPCA based approach

\begin{tabular}{ccccc}
\hline Feature(Code length) & Genuine Trials(G) & Imposter Trials (I) & Matcher(Similarity Measure) & EER\% \\
\hline 256 & 366 & 29280 & Modified & 3.0191 \\
476 & & Angle & 3.3982 \\
485 & & Distance & 3.0396 \\
500 & & & 2.2814 \\
501 & & & 2.3497 \\
714 & & & 2.4010 \\
952 & & & 3.1216 \\
\hline \multicolumn{2}{r}{}
\end{tabular}

[2] Genuine Trials $(\mathrm{G})=N * t *(t-1) / 2=366, \quad$ [3] Imposter Trials $(\mathrm{I})=N *(N-1) * t * t / 2=29280$

\subsection{Comparison with existing methods}

The performance comparison of the proposed approaches with the other existing approaches for four Iris databases are shown in Table 4 along with the feature extraction, classification techniques, and their evaluation protocols. No earlier work found based on MPCA for Iris recognition for direct performance comparison. From the previous work, it can be noticed that the deep learning approaches are generally outperformed as they are able to analyse complicated data quite well. However, deep learning-based approaches, while effective, are very computationally expensive and time-consuming [24]. Further, the training dataset size plays a large role in the creation of good feature extractors [25]. Comparing with earlier work based on WT and WPD [26]-[29], our proposed approach shows the encouraging performance among typical algorithms. MPCA utilizes fewer features while significantly improving recognition accuracy compared to Wavelet packet selection based on the Energy Measure method for feature vector formation. Thus, our method is more powerful in representing the texture features and reducing the probability of false match rate. Here, it is worth noting that very few studies used the SDUMLA-HMT Iris database for evaluating the biometric system. The feature extraction algorithm based on MPCA+LDA technique [30] can be examined in the further study of the Iris recognition problem to take into consideration the class relations [25] of the feature sets. Our prototype model ran on PC with $3.10 \mathrm{GHz}$ processor and $8 \mathrm{~GB}$ RAM. The training and testing processing time shown in Table 4 shows a significant speedup of execution.

Table 4. Comparsion of iris recognition approaches with their evaluation protocols

\begin{tabular}{|c|c|c|c|c|c|}
\hline Author & Iris Database & Method & Feature Vector & Classification & Performance Measure \\
\hline Zhiping et al. [31] & CASIA & 2D-weighted PCA & $\begin{array}{l}\text { Normalized } \\
\text { size: } 240 \times 20\end{array}$ & Adaptive ANN & $\operatorname{CRR}(\%)=97.7$ \\
\hline $\mathrm{Ng}$ et al. [26] & CASIA-Iris-V3 & Haar WT & 348 bits & Hamming & $\operatorname{CRR}(\%)=98.45$ \\
\hline Dhange et al. [28] & IITD & $\mathrm{DWT}+\mathrm{DCT}+\mathrm{BPSO}$ & $\begin{array}{l}\text { Avg.feature } \\
\text { Selected } 56\end{array}$ & Euclidean & $\operatorname{CRR}(\%)=97.81$ \\
\hline Vishi et al. [32] & SDUMLA-HMT & VeriEye $6.5 \mathrm{SDK}$ & - & - & $\mathrm{EER}=3.30 \%$ \\
\hline Kamlaskar et al. [33] & SDUMLA-HMT & 1D Log-Gabor & 9600 & Hamming & $\mathrm{EER}=2.59 \%$ \\
\hline Alay et al. [24] & SDUMLA-HMT & $\begin{array}{l}\text { VGG-16 CNN } \\
\text { model }\end{array}$ & $\begin{array}{l}\text { Feature map } \\
\text { size: } 7 \times 7 \times 512\end{array}$ & $\begin{array}{l}\text { Fully connected } \\
\text { layers: } 4096 \text { nodes }\end{array}$ & $\operatorname{Accuracy}(\%)=98.58$ \\
\hline Proposed & SDUMLA-HMT & WPD & 952 & Triangle square ratio & $\mathrm{EER}=2.298 \%$ \\
\hline
\end{tabular}

\section{CONCLUSIONS}

This paper focuses on the extraction and fusion of features of iris modality. Iris features are extracted using the WPD technique. Wavelet packet coefficients which are extracted at the 3-level, composed of approximations and details, represent iris features. Here, MPCA is proposed to consider the interrelationship among different wavelet packet coefficients and generate more discriminating features with compact representation. So, all wavelet packets are arranged in tensor format and performed a feature fusion based on a multilearning subspace learning algorithm. It aims to find transformations that preserve the multidimensional data structure, search for low-dimensional multilinear projections, and perform dimensionality reduction efficiently. These characteristics make MPCA an efficient feature fusion tool for pattern recognition. The experimental results show the efficacy of our proposed approach in the fusion of wavelet packet feature sets extracted from an iris 
modality of the SDUMLA-HMT database. Using MPCA, the best performance or the lowest EER of 2.2814\% is obtained for feature vector length of 500 compared to Wavelet packet selection based on Energy Measure method for feature vector formation. A significant contribution of this study is in significantly reducing the feature number using MPCA. Thus, making Iris recognition computationally efficient with equally high recognition accuracy. The proposed approach uses distance measure for classification, which can be further improved using advanced matching or classification methods.

\section{REFERENCES}

[1] A. K. Jain, K. Nandakumar, and A. Ross, "50 years of biometric research: Accomplishments, challenges, and opportunities," Pattern Recognition Letters, vol. 79, no. Supplement C, pp. 80-105, 2016, doi: 10.1016/j.patrec.2015.12.013.

[2] E. A. Abed, R. J. Mohammed, and D. T. Shihab, "Intelligent multimodal identification system based on local feature fusion between iris and finger vein," Indonesian Journal of Electrical Engineering and Computer Science (IJEECS), vol. 21, no. 1, pp. 224-232, 2021, doi: 10.11591/ijeecs.v21.i1.pp224-232.

[3] H. Lu, K. N. Plataniotis, and A. N. Venetsanopoulos, "MPCA: Multilinear principal component analysis of tensor objects," in IEEE Transactions on Neural Networks, vol. 19, no. 1, pp. 18-39, Jan. 2008, doi: 10.1109/TNN.2007.901277.

[4] J. Daugman, "How iris recognition works," in IEEE Transactions on Circuits and Systems for Video Technology, vol. 14, no. 1, pp. 21-30, Jan. 2004, doi: 10.1109/TCSVT.2003.818350.

[5] R. P. Wildes, "Iris recognition: an emerging biometric technology," Proceedings of the IEEE, vol. 85, no. 9, pp. 1348-1363, Sep 1997, doi: 10.1109/5.628669.

[6] W. W. Boles and B. Boashash, "A human identification technique using images of the iris and wavelet transform," IEEE Transactions on Signal Processing, vol. 46, no. 4, pp. 1185-1188, April 1998, doi: 10.1109/78.668573.

[7] Y. Zhu, T. Tan, and Y. Wang, "Biometric personal identification based on iris patterns," in Proceedings 15th International Conference on Pattern Recognition. ICPR-2000, 2000, pp. 801-804 vol. 2, doi: 10.1109/ICPR.2000.906197.

[8] S. Lim, K. Lee, O. Byeon, and T. Kim, "Efficient iris recognition through improvement of feature vector and classifier," Electronics and Telecommunications Research Institute Journal, vol. 23, no. 2, pp. 61-70, 2001, doi: 10.4218/etrij.01.0101.0203.

[9] J. Wang and M. Xie, "Iris feature extraction based on wavelet packet analysis," in 2006 International Conference on Communications, Circuits and Systems, 2006, pp. 31-34, doi: 10.1109/ICCCAS.2006.284579.

[10] S. Hariprasath and S. Venkatasubramanian, "Iris feature extraction and recognition using wavelet packet analysis," in 2010 International Conference on Signal and Image Processing, 2010, pp. 180-185, doi: 10.1109/ICSIP.2010.5697465

[11] P. S. Sanjekar and J. B. Patil, "Multimodal biometrics with serial, parallel and hierarchical mode at decision level fusion," Indonesian Journal of Electrical Engineering and Computer Science (IJEECS), vol. 16, no. 3, pp. 13031310, 2019, doi: 10.11591/ijeecs.v16.i3.pp1303-1310.

[12] K. Soumia, B. Mohammed, H. Aymen, and K. Ibrahim, "Biometric authentication using curvelet transform," Indonesian Journal of Electrical Engineering and Computer Science (IJEECS), vol. 20, no. 3, pp. 1332-1341, 2020, doi: 10.11591/ijeecs.v20i3.pp1332-1341

[13] M. A. O. Vasilescu and D. Terzopoulos, "Multilinear subspace analysis of image ensembles," in 2003 IEEE Computer Society Conference on Computer Vision and Pattern Recognition, 2003. Proceedings., 2003, pp. II-93, doi: 10.1109/CVPR.2003.1211457.

[14] H. Lu, K. N. Plataniotis, and A. N. Venetsanopoulos, "A survey of multilinear subspace learning for tensor data," Pattern Recognition, vol. 44, no. 7, pp. 1540-1551, July 2011, doi: 10.1016/j.patcog.2011.01.004.

[15] W.-J. K. Qing-Zhu Wang and Y.-J. Wang, "Support tensor machine image classification algorithm based on tensor principal component analysis," in Journal of Information Hiding and Multimedia Signal Processing, vol. 7, no. 6, pp. 1265-1273, Nov 2016

[16] R. Jillela, A. A. Ross, V. N. Boddeti, B. V. K. Vijaya Kumar, X. Hu, R. Plemmons, and P. Pauca, "Iris Segmentation for Challenging Periocular Images," Handbook of Iris Recognition, London: Springer London, pp. 281-308, 2016, doi: 10.1007/978-1-4471-6784-6_13.

[17] M. Pathak, N. Srinivasu, and V. Bairagi, "Effective segmentation of sclera, iris and pupil in eye images," TELKOMNIKA (Telecommunication Computing Electronics and Control), vol. 17, no. 5, pp. 101-111, 2019, doi: 10.12928/telkomnika.v17i5.12551.

[18] E. Rydgren, E. A. Thomas, F. Amiel, F. Rossant, and A. Amara, "Iris features extraction using wavelet packets," in 2004 International Conference on Image Processing, 2004. ICIP '04., 2004, pp. 861-864 Vol.2, doi: 10.1109/ICIP.2004.1419435.

[19] X. He, D. Cai, and P. Niyogi, “Tensor subspace analysis,” in Adv. in Neural Info. Processing Sys., pp. 499-506, 2005. 
[20] C.-C. Liu, D.-Q. Dai, and H. Yan, "Local discriminant wavelet packet coordinates for face recognition," J. Mach. Learn. Res., vol. 8, pp. 1165-1195, May 2007.

[21] Y. Yin, L. Liu, and X. Sun, "SDUMLA-HMT: A Multimodal Biometric Database," In: Sun Z., Lai J., Chen X., Tan T. (eds) Biometric Recognition. CCBR 2011. Lecture Notes in Computer Science, vol. 7098. Springer, Berlin, Heidelberg, 2011, doi: 10.1007/978-3-642-25449-9_33.

[22] Y. Lee, R. J. Micheals, J. J. Filliben, and P. J. Phillips, "VASIR: an open-source research platform for advanced iris recognition technologies," Journal of Research of the National Institute of Standards and Technology, vol. 118, pp. 218-259, 2013, doi: 10.6028/jres.118.011.

[23] L. Masek and P. Kovesi, "Matlab source code for a biometric identification system based on iris patterns," The School of Computer Science and Software Engineering, The University of Western Australia, vol. 2, no. 4, 2003.

[24] N. Alay and H. H. Al-Baity, "Deep learning approach for multimodal biometric recognition system based on fusion of iris, face, and finger vein traits," Sensors, vol. 20, no. 19, 2020, doi: 10.3390/s20195523.

[25] G. Biagetti, P. Crippa, L. Falaschetti, S. Orcioni, and C. Turchetti, "Multivariate direction scoring for dimensionality reduction in classification problems," in Intelligent Decision Technologies 2016, 2016, pp. 413-423, doi: 10.1007/978-3-319-39630-9_35.

[26] T. W. Ng, T. L. Tay, and S. W. Khor, "Iris recognition using rapid haar wavelet decomposition," in 2010 2nd International Conference on Signal Processing Systems, 2010, pp. V1-820-V1-823, doi: 10.1109/ICSPS.2010.5555246.

[27] H. Rai and A. Yadav, "Iris recognition using combined support vector machine and hamming distance approach," Expert Systems with Applications, vol. 41, no. 2, pp. 588-593, 2014, doi: 10.1016/j.eswa.2013.07.083.

[28] S. S. Dhage, S. S. Hegde, K. Manikantan, and S. Ramachandran, "DWT-based feature extraction and radon transform based contrast enhancement for improved iris recognition," Procedia Computer Science, vol. 45, pp. 256-265, 2015, doi: 10.1016/j.procs.2015.03.135.

[29] H. Ohmaid, S. Eddarouich, A. Bourouhou, and M. Timouya, "Comparison between SVM and KNN classifiers for iris recognition using a new unsupervised neural approach in segmentation," IAES International Journal of Artificial Intelligence (IJ-AI), vol. 9, no. 3, pp. 429-438, 2020, doi: 10.11591/ijai.v9.i3.pp429-438.

[30] H. Lu, K. Plataniotis, and A. N. Venetsanopoulos, "Gait recognition through mpca plus lda," in 2006 Biometrics Symposium: Special Session on Research at the Biometric Consortium Conference, 2006, pp. 1-6, doi: 10.1109/BCC.2006.4341613.

[31] Z. Zhiping, H. Maomao and S. Ziwen, "An iris recognition method based on 2dwpca and neural network," in 2009 Chinese Control and Decision Conference, 2009, pp. 2357-2360, doi: 10.1109/CCDC.2009.5192124.

[32] K. Vishi and S. Y. Yayilgan, "Multimodal biometric authentication using fingerprint and iris recognition in identity management," in 2013 Ninth International Conference on Intelligent Information Hiding and Multimedia Signal Processing, 2013, pp. 334-341, doi: 10.1109/IIH-MSP.2013.91.

[33] C. Kamlaskar, S. Deshmukh, S. Gosavi, and A. Abhyankar, "Novel canonical correlation analysis based feature level fusion algorithm for multimodal recognition in biometric sensor systems," Sensor Letters, vol. 17, no. 1, pp. 75-86, 2019, doi: $10.1166 / \mathrm{sl} .2019 .4013$.

\section{BIOGRAPHIES OF AUTHORS}

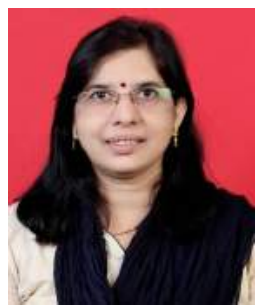

Chetana Kamlaskar is an assistant professor in School of Science and Technology at Y C M Open University, India. She received her doctoral degree PhD (E\&TC) from Savitribai Phule Pune University, formerly University of Pune (SPPU), Pune in 2019, postgraduate degree M. Tech (Communication) from IIT Bombay, Mumbai in 1998. She is member of Institution of Engineers (IE) and IETE, Life Member of ISTE. Her current research interests include Multimodal Biometrics, Machine learning, electronics, digital systems, and in eLearning systems.

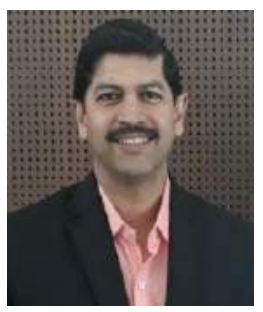

Aditya Abhyankar, Dean of the Faculty of Technology and a Professor at SP Pune University's Department of Technology. In 2001, he earned his BE in (E\&TC) from Pune University in India. In 2003 and 2006, he obtained his postgraduate degree MS and doctoral degree PhD from Clarkson University, New York, USA. Dr. Abhyankar holds US and Indian patents, and has earned a number of national and state honours. Some of his research interests include signal processing, image processing, wavelet analysis, biometric systems, and pattern recognition. 